\title{
Application of WFRFT in Impulsive Noise Channels of Substation Communications
}

\author{
Xiaolu Wang ${ }^{\dagger \ddagger}$, Lin $\mathrm{Mei}^{\dagger}$, Fabrice Labeau ${ }^{\ddagger}$ \\ ${ }^{\dagger}$ Communication Research Center, Harbin Institute of Technology, Harbin, Heilongjiang, China \\ ‡Electrical and Computer Engineering, McGill University, Montreal, Qc, Canada \\ Email: xiaolu.wang@mail.mcgill.ca, meilin@hit.edu.cn, fabrice.labeau@mcgill.ca
}

\begin{abstract}
A weighted fractional Fourier transform (WFRFT) scheme is proposed for the orthogonal frequency division multiplexing (OFDM) system to combat impulsive noise (IN) in substation communications. According to the central limit theorem, the analytic expression of the real-valued IN probability density function (PDF) after WFRFT is derived and for ease of calculation, the closed form expression of the approximate PDF is also put forward. The PDF of IN shows that WFRFT makes IN more concentrated to the small amplitudes in the fractional Fourier transform domain than in the frequency domain at the receiver. As a result, the WFRFT scheme achieves a better bit error rate (BER) performance than the conventional OFDM system. In addition, the proposed scheme is also compatible with other existing IN mitigation techniques, such as dynamic peakbased threshold estimation-lookup table (DPTE-LUT) blanking. Simulation results have verified that the derived analytic and closed form PDF expressions of IN after WFRFT and the advantage of the proposed scheme over the counterpart OFDM system in the IN channels at high SNR with and without the DPTE-LUT blanking.
\end{abstract}

\section{INTRODUCTION}

Communications inside the power substations have to confront severe degradations partly due to the high electromagnetic activity. The generated noise is mainly impulsive noise (IN) [1], which can reduce the performance and reliability of communication systems [2]. Orthogonal frequency division multiplexing (OFDM) has been utilized as a modulation scheme in the IN environment [3], since it is generally less sensitive to IN than the single carrier system. This is because the discrete Fourier transform (DFT) at the OFDM receiver spreads the IN energy over a part of symbols [4]. Nevertheless, IN can still bring about an enormous loss in the OFDM system capacity and error rate performance [5].

To reduce the adverse effect of IN, the blanking is applied at the OFDM system receiver. This method is widely used in practice because of its simple implementation and providing an improvement over the conventional OFDM system in the IN channels [6-8]. [3] first derived the closed-form expressions for signal-to-noise ratio (SNR) of the blanking output and the optimal blanking threshold, based on an assumption that some IN characteristics are available at the receiver. However, this assumptions is difficult to accomplish in practice which constrains the applicability of this method [7]. [8] proposed a method of using the estimated peak of each symbol as the blanking threshold, which was referred to as a dynamic peak-based threshold estimation-lookup table (DPTE-LUT) method. The DPTE-LUT device quantizes symbol peaks and transmits some corresponding bits to the receiver as the side information. This method is independent of the IN parameters and can achieve performance improvement of blanking relative to the conventional blanking method. Therefore, in this paper the DPTE-LUT blanking is applied to evaluate the proposed scheme relative to the OFDM scheme.

A weighted fractional Fourier transform (WFRFT)-based system is proposed for the selective fading channels in [9], which could achieve a better performance than or equal to those of OFDM and single carrier systems. Considering the symmetrical distribution of the WFRFT symbols on the timefrequency plane, it is employed to suppress narrow-band interference in [10], as well as to combat doubly selective fading [11]. Nevertheless, few works have considered to improve the communication system performance in the IN channels by leveraging features of WFRFT. Therefore, this paper is motivated threefold. First, the distribution characteristics of IN after WFRFT is analyzed theoretically. Second, WFRFT is introduced to enhance the conventional OFDM system performance in the IN channels, called WFRFT-OFDM system. Third, the contribution also resides in utilizing WFRFT with the DPTE-LUT method and increasing the quantization resolution factor.

The remainder of this paper is organized as follows. In Section II, some preliminaries about the WFRFT and the IN model are addressed. The proposed WFRFT-OFDM system and the WFRFT-OFDM system with the DPTE-LUT blanking are described in Section III. The theoretical expressions of the IN PDF after WFRFT are derived, verified and analyzed in Section IV. The simulation results that verify the proposed scheme are presented in Section V. Finally, Section VI concludes this work.

\section{PRELIMINARIES}

\section{A. Weighted fractional Fourier transform}

The $N$-point $\alpha$ order WFRFT of any complex vector $\mathbf{s}:=$ $\left[s_{0}, s_{1}, \ldots, s_{N-1}\right]^{\mathbf{t}}$ can be expressed as [9]

$$
\begin{aligned}
\mathbf{t}=\mathbf{F}^{\alpha} \mathbf{s} & =\left(\omega_{0}^{\alpha} \mathbf{I}+\omega_{1}^{\alpha} \mathbf{F}+\omega_{2}^{\alpha} \mathbf{P}+\omega_{3}^{\alpha} \mathbf{F}^{-1}\right) \mathbf{s} \\
& =\left(\omega_{0}^{\alpha} \mathbf{I}+\omega_{1}^{\alpha} \mathbf{F}+\omega_{2}^{\alpha} \mathbf{P}+\omega_{3}^{\alpha} \mathbf{P} \mathbf{F}\right) \mathbf{s}
\end{aligned}
$$

where $\mathbf{F}^{\alpha}$ is the $\alpha$ order $N \times N$ WFRFT matrix and $\mathbf{F}$ denotes the $N \times N$ unitary Fourier matrix whose entries satisfy 


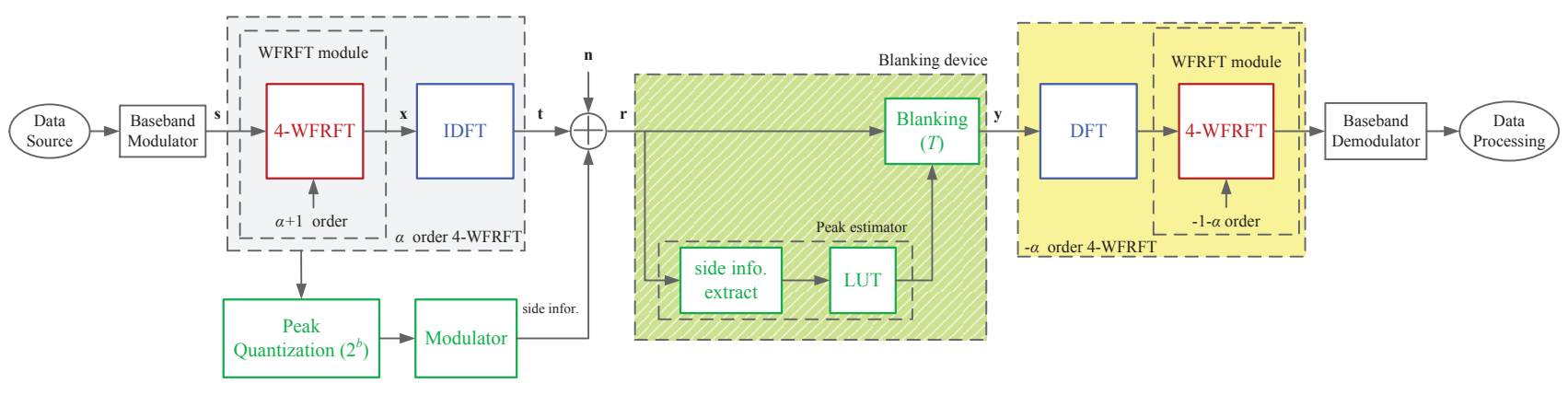

Fig. 1. Block diagram of the WFRFT-OFDM system with the DPTE-LUT blanking in the impulsive noise channel.

$[\mathbf{F}]_{k, m}:=(1 / \sqrt{N}) \exp (-j 2 \pi k m / N)$, where $[\cdot]_{k, m}(k, m=$ $0,1, \ldots N-1)$ indicates the specific element located at $k$-th row and $m$-th column in a matrix. $\mathbf{I}$ is the identity matrix and the weighting coefficients $\omega_{l}^{\alpha}(l=0,1,2,3)$ are given by [9]

$$
\omega_{l}^{\alpha}=\frac{1}{4} \sum_{m=0}^{3} \exp \left[\frac{\mathrm{j} 2 \pi(\alpha-l) m}{4}\right] \quad(l=0,1,2,3)
$$

As $\omega$ is periodic, i.e. $\omega_{l}^{\alpha}=\omega_{l}^{\alpha+4}$, and $\alpha$ is a real number, the main interval of $\alpha$ is always chosen in [-2,2] or [0,4].

The shift matrix $\mathbf{P}$ satisfies $[\mathbf{P}]_{k, m}=\delta\left(\langle k+m\rangle_{N}\right)$, where $\langle\cdot\rangle_{N}$ denotes modulo- $N$ calculation [10]. $\mathbf{F}^{\alpha}$ is a unitary matrix which satisfies

$$
\mathbf{F}^{-\alpha}=\left[\mathbf{F}^{\alpha}\right]^{-1}=\left[\mathbf{F}^{\alpha}\right]^{\mathrm{H}}
$$

where $\left[\mathbf{F}^{\alpha}\right]^{-1}$ is the inverse matrix of $\mathbf{F}^{\alpha}$, and $\left[\mathbf{F}^{\alpha}\right]^{\mathrm{H}}$ is the conjugate transposed matrix of $\mathbf{F}^{\alpha}$. It can be obtained that $\mathbf{F}^{0}=\mathbf{I}$ and $\mathbf{F}^{1}=\mathbf{F}$. And there is an important Additive Axiom:

$$
\mathbf{F}^{\alpha+\beta}=\mathbf{F}^{\alpha} \mathbf{F}^{\beta}=\mathbf{F}^{\beta} \mathbf{F}^{\alpha}
$$

\section{B. Impulsive noise model}

In this paper, the Middleton Class A impulsive noise model [12] is used to evaluate the system performance. This model has been found to describe a wide variety of electromagnetic noise, including the power line and the electric tool emissions which were found to match the measured IN distributions well [12].

The probability density function (PDF) of the real-valued Class A noise $n$ is given by [12]

$$
f_{A}(n)=\sum_{m=0}^{\infty} \frac{g_{m}}{\sigma_{m} \sqrt{2 \pi}} \exp \left(-\frac{n^{2}}{2 \sigma_{m}^{2}}\right)
$$

with

$$
g_{m}=\frac{e^{-A} A^{m}}{m !} \quad \text { and } \quad \sigma_{m}^{2}=\sigma^{2} \frac{m / A+\Gamma}{1+\Gamma}
$$

where $A$ denotes the product between the impulsive rate, i.e. the average number of impulses per time unit, and the impulse mean duration. In other words, the higher $A$ is, the more impulse events there are and the more similar the noise is to the Gaussian noise. $\Gamma$ presents the Gaussian to impulsive noise power ratio (GIR) which is the ratio between the average power of the additive white Gaussian noise (AWGN) component $\sigma_{G}^{2}$ and the IN component $\sigma_{I}^{2} \cdot \sigma^{2}=\sigma_{G}^{2}+\sigma_{I}^{2}$ is the total noise power. When the parameter $A$ is fixed and $\Gamma$ is lower, the IN is more harmful. Here, SNR is defined as $10 \log _{10}\left(\sigma_{a}^{2} / \sigma_{G}^{2}\right)$ [6-8], where $\sigma_{a}^{2}$ is the average power of complex baseband signals.

\section{SYSTEM MODELS}

The block diagram of the WFRFT-OFDM system with the DPTE-LUT blanking is depicted in Fig.1, where the WFRFT modules are placed before/after IDFT/DFT of a conventional OFDM system. The DPTE-LUT blanking [8] is placed before the $(-\alpha)$ order WFRFT module at the receiver. When the peak quantization block and the blanking device are removed from the transmitter and receiver, it is referred to as a WFRFTOFDM system. Between the DFT/IDFT and WFRFT modules, there might be some other operations, such as frequency mapping or frequency domain equalization, which are not utilized and displayed in this paper.

The main idea of IN suppression using WFRFT is that WFRFT can change the IN probability distribution leading to a better BER performance than the conventional OFDM at the receiver. Meanwhile, WFRFT can be easily implemented by DFT, which has the same complexity as fast Fourier transform (FFT). A 16QAM vector $\mathbf{s}$ is assumed to be in the $(-\alpha)$ order WFRFT domain, which can be converted into time domain through $\alpha$ order WFRFT. According to the Additive Axiom shown in (4), the transmitted WFRFT-OFDM symbols can be expressed as:

$$
\mathbf{t}=\mathbf{F}^{-1} \mathbf{F}^{\alpha+1} \mathbf{s}=\mathbf{F}^{-1} \mathbf{x}=\mathbf{F}^{\alpha} \mathbf{s}
$$

At the transmitter, every symbol envelope peak is uniformly quantized into a finite number of levels, where the levels are determined by a predetermined minimum value $P_{\text {min }}$, a maximum value $P_{\max }$ and the number of bits representing each symbol envelope peak. The resolution factor $R_{F}$, which is the spacing between quantization levels, is defined as

$$
R_{F}=\frac{P_{\max }-P_{\min }}{N_{q}-1}
$$

where $N_{q}=2^{b}$ and $b$ is the number of the quantization bit. Here, $b$ is set to 5 [8]. The dynamic range of the transmitted symbol peaks determines $P_{\min }$ and $P_{\max }$. When $b$ is fixed, a 
smaller dynamic range will get a higher resolution factor. The $b$ bits which represent the quantized peaks are transmitted to the receiver as some side information with each WFRFT-OFDM symbol using 16QAM modulation [see [8] for more details].

With perfect synchronization, the received signals can be expressed as

$$
\mathbf{r}=\mathbf{t}+\mathbf{n}
$$

where $\mathbf{n}$ denotes the non-Gaussian noise which contains AWGN and IN.

At the receiver, blanking is applied before a baseband demodulator. The basic principle of a blanking device follows [6]:

$$
y_{k}=\left\{\begin{array}{cl}
r_{k}, & \left|r_{k}\right| \leq T \\
0, & \left|r_{k}\right|>T
\end{array} \quad k=0,1, \ldots, N-1\right.
$$

where $T$ denotes the blanking threshold. $r_{k}$ and $y_{k}$ are the input and output of the blanking device, respectively. If $T$ is very large, blanking processing will have a negligible effect on the received baseband signals as a result of allowing most of the IN to pass the blanking device. On the other hand, for very small $T$, most of the received samples of the baseband signals will be set to zero causing poor BER performance. Therefore, the blanking threshold must be carefully selected. Here, the DPTE-LUT based algorithm with uniform quantization is used for blanking threshold choosing. The estimated symbol envelope peak based on the side information is taken as its blanking threshold. The basic principle of the blanking according to DPTE-LUT method is [8]

$$
y_{k}=\left\{\begin{array}{rl}
r_{k}, & \left|r_{k}\right| \leq \tilde{P}_{e} \\
0, & \left|r_{k}\right|>\tilde{P}_{e}
\end{array} \quad k=0,1, \ldots, N-1\right.
$$

where $\tilde{P}_{e}$ is the estimated symbol peak value based on the side information at the receiver. Then, the blanked signals $\mathbf{y}$ are processed by DFT and $(-1-\alpha)$ order WFRFT at the receiver, which equals to the processing of $(-\alpha)$ order WFRFT.

\section{Probability Characteristics of IMPUlsive NOSIE AFTER WFRFT}

The probability characteristics of non-Gaussian noise influence the signal demodulation performance at the receiver significantly. WFRFT can change the PDF of IN, so a PDF analytic expression of IN after WFRFT in the WFRFT-OFDM system is obtained to show the IN PDF variations compared to the OFDM system. The IN after $(-\alpha)$ order WFRFT at the receiver can be expressed as

$$
\tilde{\mathbf{n}}=\mathbf{F}^{-\alpha} \mathbf{n}=\omega_{0}^{-\alpha} \mathbf{I n}+\omega_{1}^{-\alpha} \mathbf{F n}+\omega_{2}^{-\alpha} \mathbf{P n}+\omega_{3}^{-\alpha} \mathbf{F}^{-1} \mathbf{n}
$$

As the real and imaginary parts of $\tilde{\mathbf{n}}$ have the same PDF, the real part of $\tilde{\mathbf{n}}$ is analyzed as an example, i.e., $\Re[\tilde{\mathbf{n}}]=$ $\Re\left[\omega_{0}^{-\alpha} \mathbf{I n}\right]+\Re\left[\omega_{1}^{-\alpha} \mathbf{F n}\right]+\Re\left[\omega_{2}^{-\alpha} \mathbf{P n}\right]+\Re\left[\omega_{3}^{-\alpha} \mathbf{F}^{-1} \mathbf{n}\right]$.

Since the elements of the vector $\Re[\tilde{\mathbf{n}}]$ are mutually independent and have the same PDF, any element of $\Re[\tilde{\mathbf{n}}]$ can be chosen to show the distribution characteristics of IN after WFRFT. Hence, $\mathscr{E}\{\cdot\}$ is used to denote the first element of a vector and the second part $\mathscr{E}\left\{\Re\left[\omega_{1}^{-\alpha} \mathbf{F n}\right]\right\}$ of $\mathscr{E}\{\Re[\tilde{\mathbf{n}}]\}$ can be written as

$$
\mathscr{E}\left\{\Re\left[\omega_{1}^{-\alpha} \mathbf{F n}\right]\right\}=\mathscr{E}\left\{\Re\left[\omega_{1}^{-\alpha}\right] \Re[\mathbf{F n}]\right\}-\mathscr{E}\left\{\Im\left[\omega_{1}^{-\alpha}\right] \Im[\mathbf{F n}]\right\}
$$

where $\Re[\cdot]$ and $\Im[\cdot]$ mean the real part and the imaginary part. According to the Central Limit Theorem, $\mathscr{E}\left\{\Re\left[\omega_{1}^{\alpha}\right] \Re[\mathbf{F n}]\right\}$ and $\mathscr{E}\left\{-\Im\left[\omega_{1}^{\alpha}\right] \Im[\mathbf{F n}]\right\}$ follow $N\left(0,\left[\Re\left[\omega_{1}^{\alpha}\right]\right]^{2} \sigma^{2}\right)$ and $N\left(0,\left[\Im\left[\omega_{1}^{\alpha}\right]\right]^{2} \sigma^{2}\right)$. The sum of these two independent normally distributed random variables follows $N\left(0,\left|\omega_{1}^{-\alpha}\right|^{2} \sigma^{2}\right)$ [13].

The same rules also applies in $\mathscr{E}\left\{\Re\left[\omega_{3}^{-\alpha} \mathbf{F}^{-1} \mathbf{n}\right]\right\}$ and its distribution is $N\left(0,\left|\omega_{3}^{-\alpha}\right|^{2} \sigma^{2}\right)$. Thus, it is obtained that

$\mathscr{E}\left\{\Re\left[\omega_{1}^{-\alpha} \mathbf{F n}\right]+\Re\left[\omega_{3}^{-\alpha} \mathbf{F}^{-1} \mathbf{n}\right]\right\} \sim N\left(0,\left(\left|\omega_{1}^{-\alpha}\right|^{2}+\left|\omega_{3}^{-\alpha}\right|^{2}\right) \sigma^{2}\right)$

The first part $\mathscr{E}\left\{\Re\left[\omega_{0}^{-\alpha} \mathbf{I n}\right]\right\}$ of $\mathscr{E}\{\Re[\tilde{\mathbf{n}}]\}$ is

$$
\mathscr{E}\left\{\Re\left[\omega_{0}^{-\alpha} \mathbf{I n}\right]\right\}=\mathscr{E}\left\{\Re\left[\omega_{0}^{-\alpha}\right] \Re[\mathbf{I n}]\right\}-\mathscr{E}\left\{\Im\left[\omega_{0}^{-\alpha}\right] \Im[\mathbf{I n}]\right\}
$$

where $\mathscr{E}\left\{\Re\left[\omega_{0}^{-\alpha}\right] \Re[\mathbf{I n}]\right\}$ and $\mathscr{E}\left\{-\Im\left[\omega_{0}^{-\alpha}\right] \Im[\mathbf{I n}]\right\}$ are two independent real-valued IN variables based on (5) and the distribution of the sum of these two independent variables can be calculated through convolution [13]. After some convolution calculations, the PDF of $\mathscr{E}\left\{\Re\left[\omega_{0}^{-\alpha} \mathbf{I n}\right]\right\}$ is formulated as

$$
\begin{array}{r}
f_{x}=\sum_{p=0}^{\infty} \sum_{q=0}^{\infty} \frac{g_{p} g_{q}}{\sqrt{2 \pi\left(\sigma_{0 p}^{2}+\sigma_{0 q}^{2}\right)}} \exp \left[-\frac{x^{2}}{2\left(\sigma_{0 p}^{2}+\sigma_{0 q}^{2}\right)}\right] \\
\text { where } \sigma_{0 p}^{2}=\left[\Re\left[\omega_{0}^{-\alpha}\right]\right]^{2} \sigma^{2} \frac{p / A+\Gamma}{1+\Gamma}, g_{p}=\frac{e^{-A} A^{p}}{p !}, \\
\sigma_{0 q}^{2}=\left[\Im\left[\omega_{0}^{-\alpha}\right]\right]^{2} \sigma^{2} \frac{q / A+\Gamma}{1+\Gamma}, g_{q}=\frac{e^{-A} A^{q}}{q !}
\end{array}
$$

Similarly, the PDF of $\mathscr{E}\left\{\Re\left[\omega_{2}^{-\alpha} \mathbf{P n}\right]\right\}$ is formulated as

$$
\begin{array}{r}
f_{y}=\sum_{s=0}^{\infty} \sum_{k=0}^{\infty} \frac{g_{s} g_{k}}{\sqrt{2 \pi\left(\sigma_{2 s}^{2}+\sigma_{2 k}^{2}\right)}} \exp \left[-\frac{y^{2}}{2\left(\sigma_{2 s}^{2}+\sigma_{2 k}^{2}\right)}\right] \\
\text { where } \sigma_{2 s}^{2}=\left[\Re\left[\omega_{2}^{-\alpha}\right]\right]^{2} \sigma^{2} \frac{s / A+\Gamma}{1+\Gamma}, g_{s}=\frac{e^{-A} A^{s}}{s !}, \\
\sigma_{2 k}^{2}=\left[\Im\left[\omega_{2}^{-\alpha}\right]\right]^{2} \sigma^{2} \frac{k / A+\Gamma}{1+\Gamma}, g_{k}=\frac{e^{-A} A^{k}}{k !}
\end{array}
$$

Now it can be seen that $\mathscr{E}\{\Re[\tilde{\mathbf{n}}]\}$ is the sum of three mutually independent random variables, i.e., $\mathscr{E}\left\{\Re\left[\omega_{1}^{-\alpha} \mathbf{F n}\right]+\right.$

$$
f_{\tilde{n}_{r}}=\sum_{p=0}^{\infty} \sum_{q=0}^{\infty} \sum_{s=0}^{\infty} \sum_{k=0}^{\infty} \frac{g_{p} g_{q} g_{s} g_{k}}{\sqrt{2 \pi} \sqrt{\sigma_{0 p}^{2}+\sigma_{0 q}^{2}+\sigma_{2 s}^{2}+\sigma_{2 k}^{2}+\left(\left|\omega_{1}^{2}\right|^{2}+\left|\omega_{3}^{2}\right|^{2}\right) \sigma^{2}}} \exp \left(-\frac{\tilde{n}_{r}^{2}}{2\left[\sigma_{0 p}^{2}+\sigma_{0 q}^{2}+\sigma_{2 s}^{2}+\sigma_{2 k}^{2}+\left(\left|\omega_{1}^{2}\right|^{2}+\left|\omega_{3}^{2}\right|^{2}\right) \sigma^{2}\right]}\right)
$$




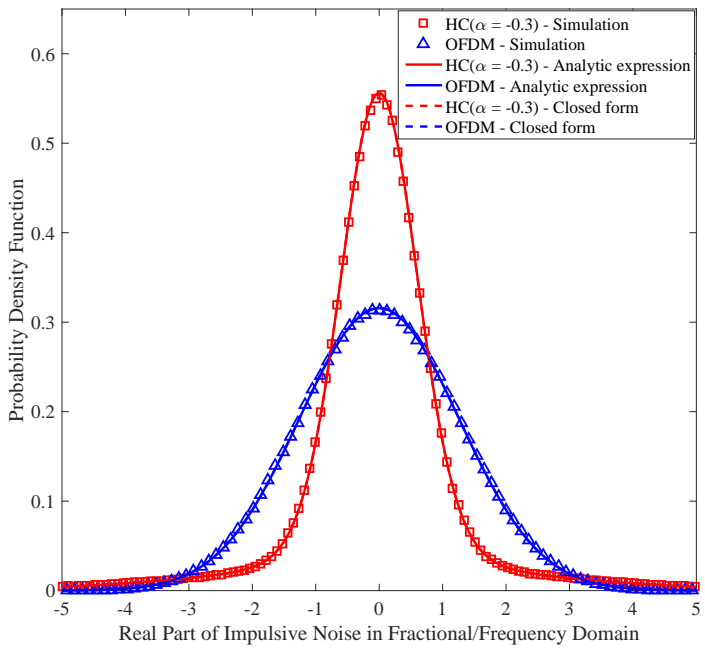

Fig. 2. PDF of the real part of impulsive noise in fractional/frequency domain for $16 \mathrm{QAM}$ in $A=0.1, \Gamma=0.01$.

$\left.\Re\left[\omega_{3}^{-\alpha} \mathbf{F}^{-1} \mathbf{n}\right]\right\}, \mathscr{E}\left\{\Re\left[\omega_{0}^{-\alpha} \mathbf{I n}\right]\right\}$ and $\mathscr{E}\left\{\Re\left[\omega_{2}^{-\alpha} \mathbf{P n}\right]\right\}$. Therefore, based on (12), (13) and (14), the PDF $f_{\tilde{n} r}$ of the sum of these three mutually independent random variables can be obtained through some mathematical calculations [13]. The analytic expression of real-valued $\mathscr{E}\{\Re[\tilde{\mathbf{n}}]\}$ PDF is shown as (15). For ease of calculation, the closed form expression of $\mathscr{E}\{\Re[\tilde{\mathbf{n}}]\}$ approximate PDF can also be gained when replacing $p, q, s, k=\{0,1,2 \cdots \infty\}$ in (15) with $p, q, s, k=\{0,1,2\}$.

When $\alpha=-1$, the WFRFT-OFDM system is consistent with the OFDM system which implies that the PDF expression (15) can also show the PDF of IN in frequency domain. Fig.2 is the PDF of the real part of IN at the receiver in fractional/frequency domain. It is shown that the curves of the analytic and closed form expressions coincide with each other and both match well with the simulation results in the fractional/frequency domain. Compared with the OFDM processing (only DFT at the receiver), the PDF of the IN after WFRFT $(\alpha=-0.3)$ is more concentrated to the small amplitudes [-0.8,0.8]. In the intermediate amplitude range $[0.8,3.2] \cup[-3.2,-0.8]$, there is less IN in the fractional domain. Consequently, WFRFT can improve the conventional OFDM system demodulation performance through reducing the intermediate amplitude IN occurrence probability and increasing the small amplitude IN occurrence probability. However, it is also noticed that in the high amplitude range $[-\infty, 3.2] \cup[3.2,+\infty]$ there is slightly more IN in the fractional domain than those in the frequency domain. Therefore, eliminating high amplitude IN in the fractional domain will be considered in the future research to further develop WFRFTOFDM scheme.

\section{Simulation And Analysis}

This section verifies the advantage of the WFRFT-OFDM system (referred to as WFRFT system). -0.3 is chosen as the order of WFRFT (the WFRFT system in $\alpha \in(-1,0)$ all can obtain better performance than the OFDM system and
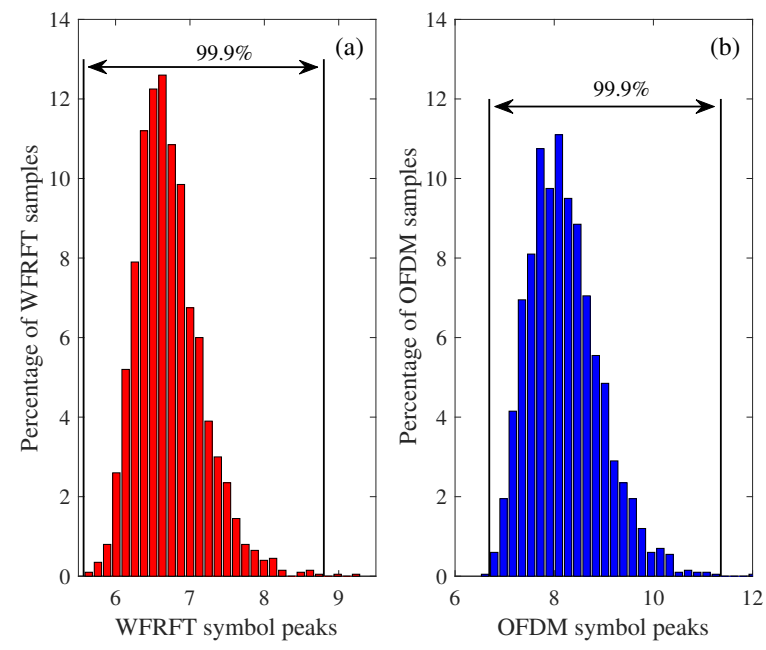

Fig. 3. Symbol peak distribution for 16QAM: (a) WFRFT ( $\alpha=-0.3)$; (b) OFDM

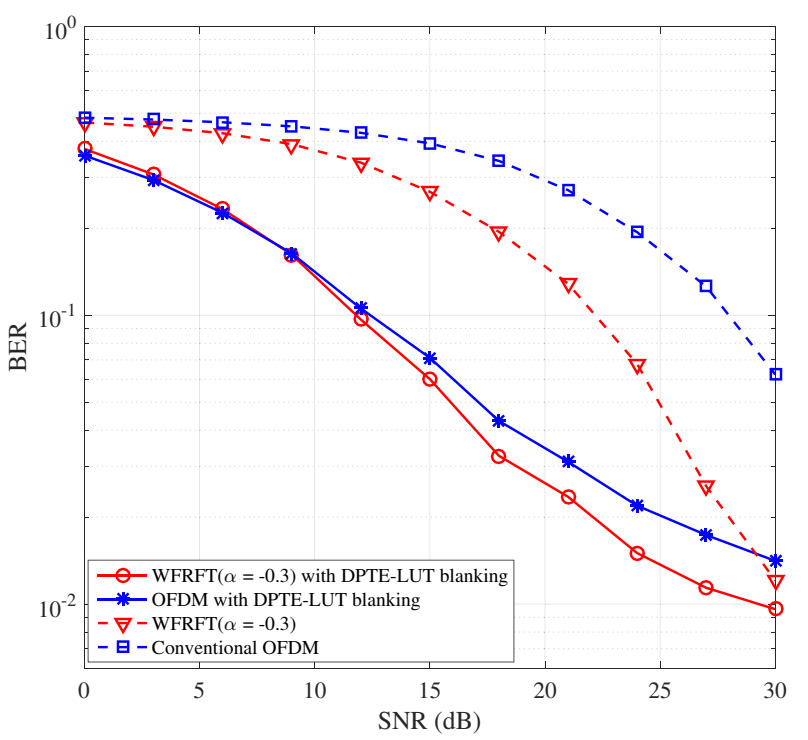

Fig. 4. BER versus SNR for WFRFT-OFDM and OFDM systems with the DPTE-LUT blanking for 16QAM in $A=0.01$ and $\Gamma=0.01$.

the optimal order of the WFRFT system is beyond the scope of this paper). The simulations are based on the parameters consisting of $N=512$ subcarriers with 16QAM modulation and 5 bit quantization in DPTE-LUT. Ideal DPTE-LUT is assumed, which means the quantized signal peaks are detected at the receiver error-free.

The simulations are conducted to contrast the BER performance of the WFRFT-OFDM and the conventional OFDM systems without blanking in the IN scenario $(A=0.01$, $\Gamma=0.01$ ). The dotted lines in Fig.4 show the resulting BER as a function of SNR for the WFRFT and conventional OFDM systems. It can be seen that the BER performance of WFRFT is better than the conventional OFDM's. When BER equals to 0.1 , the gain of $5.5 \mathrm{~dB}$ can be obtained.

For using the DPTE-LUT method, the signal peak dis- 
tribution should be applied to determine the quantification range (i.e. determine $P_{\min }$ and $P_{\max }$ ). The bar-charts for the signal envelope peaks distribution of WFRFT and OFDM are depicted in Fig.3. It is shown that the ranges, where $99.9 \%$ of the symbol envelope peaks are included, are [5.57, 8.80] and $[6.69,11.36]$ for WFRFT and OFDM respectively. As mentioned above, WFRFT has the feature which makes the PDF of signals more concentrated. Therefore, when the quantization level number is fixed, the resolution factor of WFRFT is higher than OFDM, which can reduce the quantization noise and improve the system performance. For 16QAM and 512 subcarriers, according to (7) the resolution factor in OFDM is $44.58 \%$ lower than that in WFRFT.

Fig.4 also shows the BER performance for the WFRFTOFDM and OFDM systems with the DPTE-LUT blanking. It is shown that the DPTE-LUT blanking technique can improve the system BER performance greatly. Moreover, the WFRFTOFDM system with the DPTE-LUT blanking outperforms the OFDM system with the DPTE-LUT blanking when SNR is larger than $9 \mathrm{~dB}$. From another perspective, WFRFT can enhance the blanking based on the DPTE-LUT method for the OFDM system in the IN channels. Simulation results in different IN channels are also obtained and they also verify that WFRFT can enhance the OFDM system performance.

\section{CONCLUSION}

In this paper, WFRFT is first introduced in the IN channels of substation communications. Through the theoretical analysis of IN PDF after WFRFT, the real-valued analytic and closed form PDF expressions of IN after WFRFT are obtained and they can agree well with the simulation results. It is demonstrated that WFRFT can change the distribution of IN to be more concentrated to the small amplitudes than OFDM does. The simulation results also indicate that the WFRFTOFDM system can achieve better BER performance than the counterpart conventional OFDM system in the IN channels. Furthermore, WFRFT is also evaluated in a system with the DPTE-LUT blanking. WFRFT can improve the quantization resolution factor of the DPTE-LUT method. The resolution factor in OFDM is $44.58 \%$ lower than that in WFRFT ( $\alpha=-0.3$ ) for 16QAM and 512 subcarriers. The WFRFTOFDM system with the DPTE-LUT blanking also outperforms the OFDM system with the DPTE-LUT blanking in the IN channels at high SNR. In the future research, we will focus on the channel coding to improve the WFRFT-OFDM system transmission performance.

\section{ACKNOWLEDGMENT}

This work was supported by Hydro-Qubec, the Natural Sciences and Engineering Research Council of Canada and McGill University in the framework of the NSERC/HydroQubec/McGill Industrial Research Chair in Interactive Information Infrastructure for the Power Grid (Project IRCPJ 406021-14), the National Natural Science Foundation Program of China (No.61201146), the National Basic Research
Program of China (2013CB329003) and China Scholarship Council.

\section{REFERENCES}

[1] Q. Shan, I. A. Glover, R. C. Atkinson, S. A. Bhatti, I. E. Portugues, P. J. Moore, R. Rutherford, M. de Fatima Queiroz Vieira, A. M. N. Lima, and B. A. De Souza, "Estimation of impulsive noise in an electricity substation," IEEE Trans. Electromagn. Compat., vol. 53, no. 3, pp. 653-663, 2011.

[2] M. G. Sánchez, I. Cuinas, and A. V. Alejos, "Interference and impairments in radio communication systems due to industrial shot noise," in Industrial Electronics, 2007. ISIE 2007. IEEE International Symposium on. IEEE, 2007, pp. 1849-1854.

[3] S. V. Zhidkov, "Performance analysis and optimization of OFDM receiver with blanking nonlinearity in impulsive noise environment," IEEE Veh. Technol. Mag., vol. 55, no. 1, pp. 234-242, 2006.

[4] J. A. Bingham, "Multicarrier modulation for data transmission: An idea whose time has come," IEEE Commun. Mag., vol. 28, no. 5, pp. 5-14, 1990.

[5] J. Haring and H. Vinck, "OFDM transmission corrupted by impulsive noise," in 4th International Symposium on Power-Line Communications and its Applications (ISPLC2000), 2000.

[6] S. V. Zhidkov, "Analysis and comparison of several simple impulsive noise mitigation schemes for OFDM receivers," IEEE Trans. Commun., vol. 56, no. 1, pp. 59, 2008.

[7] E. Alsusa and K. M. Rabie, "Dynamic peak-based threshold estimation method for mitigating impulsive noise in power-line communication systems," IEEE Trans. Power Del., vol. 28, no. 4, pp. 2201-2208, 2013.

[8] K. M. Rabie and E. Alsusa, "Quantized peak-based impulsive noise blanking in power-line communications," IEEE Trans. Power Del., vol. 29, no. 4, pp. 1630-1638, 2014.

[9] L. Mei, X.-j. Sha, and N.-t. Zhang, "The approach to carrier scheme convergence based on 4-weighted fractional fourier transform," IEEE Commun. Lett., vol. 14, no. 6, pp. 503-505, 2010.

[10] L. Mei, Q. Zhang, X. Sha, and N. Zhang, "WFRFT precoding for narrowband interference suppression in DFT-based block transmission systems," IEEE Commun. Lett., vol. 17, no. 10, pp. 1916-1919, 2013.

[11] K. Wang, X. Sha, and L. Mei, "On interference suppression in doubly-dispersive channels with hybrid singlemulti carrier modulation and an MMSE iterative equalizer," IEEE Commun. Lett., vol. 1, no. 5, pp. 504-507, 2012.

[12] D. Middleton, "Statistical-physical models of electromagnetic interference," IEEE Trans. Electromagn. Compat., no. 3, pp. 106-127, 1977.

[13] M. Taboga, Lectures on probability theory and mathematical statistics. CreateSpace Independent Pub., 2012. 